\title{
Ethics in EMS and Disaster Research
}

\author{
Samuel J. Stratton, MD, MPH
}

The advancement of emergency medical services (EMS) and disaster medicine requires vigorous research. Without systematic investigation, the knowledge base for EMS and disaster medicine will fail to develop and advance. Adding to the scientific knowledge base should be the goal of a researcher, and writing only for personal advancement and acclaim are to be discouraged. It is important that researchers maintain high ethical standards and avoid conflicting interests when participating in research projects.

Researchers are responsible for conducting ethical studies. This includes gathering data in a meticulous manner, keeping study databases with protection of subject privacy, and interpreting results objectively and without bias. Studies must be designed to limit potential bias. In addition, a study should be designed such that another researcher can reproduce the study and validate the findings. Conclusions should be limited to study findings based on the data and evidence presented in the research. Above all, any research study must adhere to the highest ethics with respect to human subjects.

Ethical EMS and disaster medical research can be challenging because study populations are often suffering a personal or community crisis and are therefore vulnerable to exploitation. It is important that, when appropriate, an informed consent process be in place to enter subjects into a study, and that for all forms of human research, there are adequate personal rights and confidentiality protections. Most important is that the well-being of each research subject takes precedence over all other interests. ${ }^{1}$

Prehospital and Disaster Medicine requires that all submitted research papers undergo independent review for compliance with ethical standards in conducting human subjects research. Authors most often accomplish this task by submitting research protocols to human subjects ethics committees or institutional review boards for review and approval of adherence to ethical research standards. As befitting an international journal, ethical principles for medical research involving human subjects described in the Declaration of Helsinki ${ }^{1}$ are considered the standard for Prehospital and Disaster Medicine. The original version of the Declaration was published in 1964, with eight subsequent revisions. The current version was published in 2008. Among the 35 principles stated in the Declaration of Helsinki,

References

1. World Medical Association. Declaration of Helsinki - Ethical Principles for Medical Research Involving Human Subjects. http://www.wma.net/en/30publications/10policies/ b3/index.html. Updated 2008. Accessed September 21, 2012. the following are particularly relevant to EMS and disaster medical research:

- "It is a duty in medical research to protect the life, health, dignity, integrity, right to self-determination, privacy, and confidentiality of personal information of research subjects."

- "The research must be submitted for consideration and approval to a research ethics committee before the study begins."

- "Medical research involving a vulnerable population or community is only justified if the research is responsive to the health needs and priorities of the population or community and if there is a reasonable likelihood that this population or community stands to benefit from the results of the research."

- "Every precaution must be taken to protect the privacy of research subjects and the confidentiality of their personal information and to minimize the impact of the study on their physical, mental, and social integrity."

- "Authors, editors and publishers all have ethical obligations with regard to the publication of the results of research." "Reports of research not in accordance with the principles of this Declaration should not be accepted for publication."

The Declaration of Helsinki is an accepted standard for publishing papers in almost all reputable journals of international scope. Authors and readers of Prehospital and Disaster Medicine are encouraged to review the 35 principles of the Declaration, which are concise and readily available in electronic format. ${ }^{1}$

While authors associated with university or health ministry programs are usually able to access an ethics committee, there are situations in which an author may not have access to independent ethics review. Because Prehospital and Disaster Medicine accepts and publishes papers from all areas of the world, the editorial staff is sensitive to situations in which authors may not have access to ethics committees for review of their work. In these situations, researchers are encouraged to contact the Editorial Office prior to submission of their work for advice and direction regarding compliance with the Declaration of Helsinki. 